\title{
Hemophagocytic lymphohistiocytosis as an unexpected complication of Venetoclax+Azacitidine in Acute Myeloid Leukemia
}

\begin{abstract}
Background: Venetoclax is a drug that targets BCL-2 protein in cancer cells, first approved for chronic lymphocytic leukemia, this drug has showed efficacy also in acute myeloid leukemia in non-intense chemotherapy candidates in combination with hypomethylating agents as azacitidine and decitabine. This scheme has shown efficiency in acute myeloid leukemia reporting overall response rate (CR) in $61 \%$ in untreated elderly patients combined with azacitidine or decitabine. Febrile neutropenia was reported in 30\%, thrombocytopenia in $47 \%$, and serious infections in $33 \%$. Hemophagocytic lymphohistiocytosis (HLH) is an uncommon hematologic disorder caused by a proinflammatory state manifested by cytopenias and elevation of acute phase reactants; it is a severe complication of some diseases and to our knowledge it has never been reported secondary to venetoclax plus azacitidine. Early treatment is fundamental for success in HLH.
\end{abstract}

Case series: Three cases of HLH secondary to venetoclax plus azacitidine have appeared in our medical group in patients treated for acute myeloid leukemia. One elderly woman and elderly men with previously untreated acute myeloid leukemia presented HLH with laboratory and bone marrow findings, both responded to dexamethasone plus ruxolitinib. The third case was documented in a male diagnosed with blast phase chronic myeloid leukemia who also responded to dexamethasone plus ruxolitinib. No patient died from HLH.

Conclusion: Here we report three cases of patients with HLH after the treatment with azacitidine plus venetoclax. We suspect that the great effect of venetoclax in synergy with azacitidine can liberate enough proinflammatory cytokines in the medullar niche to induce HLH. Early recognition is vital for soon treatment and successful management of this potential complication

Keywords: hemophagocytic lymphohistiocytosis, acute myeloid leukemia, 5-azacitidine, venetoclax, ruxolitinib
Volume 9 Issue I - 202 |

\author{
Ovilla-Martinez Roberto,' Perez-Lozano \\ Uendy,' Cota-Rangel Xochitl,' Baez-Islas \\ Pamela' \\ 'Hospital Ángeles Lomas, Vialidad de la Barranca s/n. \\ Huixquilucan, México \\ ${ }^{2}$ Hospital Ángeles Puebla, Reserva Territorial Atlixcayotl, México
}

Correspondence: Roberto Ovilla Martinez, MD, Box 830, Hospital Angeles Lomas, Vialidad de la Barranca, 52787, Huixquilucan, Edo. Mex, Mexico, Tel +52-55-5246-9424, Email ovillarob@gmail.com

Received: January 28, 202I | Published: February 26, 2021

\section{Introduction}

In this article we aspire to report an unusual complication of the azacitidine + venetoclax scheme used in patients with acute myeloid leukemia. Venetoclax is a BCL-2 inhibitor FDA-approved for chronic lymphocytic leukemia and some B-cell no Hodgkin lymphomas due to its high efficiency in this kind of tumors. ${ }^{1-3}$ Lately, this BCL-2 inhibitor has shown efficiency in AML. A phase 1b study published in January 2018 demonstrated that, in untreated elderly patients (ages $>65$ years old) diagnosed with AML treated with venetoclax with azacytidine or decitabine, complete remission was reached in $61 \%$ with an overall survival of 14.2 months. ${ }^{4}$ Serious adverse events observed were febrile neutropenia in $30 \%$, thrombocytopenia in $47 \%$, and serious infections in $33 \%$, mostly on patients with high dose of venetoclax and those with decitabine as hypomethylating agent. ${ }^{4}$ This study supported the FDA approval for venetoclax in the treatment of AML. Hospital Angeles is a medical group with several hospitals in Mexico. From the patients treated with this scheme in our medical group we have observed hemophagocytic lymphohistocytosis (HLH) in 3 patients, an adverse reaction that to our knowledge hasn't been described before. Here we present three cases, patients 1 and 2 with "de novo" AML from Angeles Lomas in Mexico City and patient 3 with secondary AML from Angeles Puebla.

\section{Case I}

Sixty seven year old female with medical history of high blood pressure was admitted due to fever and anemia. At admission she had these results: $\mathrm{Hb} 9.3 \mathrm{~g} / \mathrm{dl}$, WBC $3.9 \times 10^{3} / \propto 1$, granulocytes $0.6 \times 10^{3} / \propto 1$, platelets $47 \times 10^{3} / \propto 1$, myeloid blasts $15 \%$. Bone marrow aspirate had $63 \%$ blasts with AML morphology. Cytogenetic risk was intermediate due to trisomy 8 and negative mutational panel for AML. Because of her age and general status venetoclax + azacytidine scheme (venetoclax starting at $20 \mathrm{mg} /$ day in ramp-up to $400 \mathrm{mg} /$ day, azacytidine $50 \mathrm{mg} / \mathrm{m} 2$ for ten days) was started. She presented multisegmental pneumonia that required mechanical ventilation and wide spectrum antibiotics. On the $11^{\text {th }}$ day of treatment, venetoclax was suspended due to profound cytopenia. On the $21^{\text {st }}$ day of treatment a bone marrow aspirate showed aplasia and histocytes. She continued with profound pancytopenia and in day +27 a new bone marrow aspirate showed abundant hemophagocytes. In blood she had: $\mathrm{Hb}$ $8.6 \mathrm{~g} / \mathrm{dl}$, WBC $0.4 \times 10^{3} / \propto 1$, granulocytes $0 \times 10^{3} / \propto 1$, platelets $17 \times 10^{3} / \propto 1$, ferritin $2624 \mathrm{ug} / \mathrm{l}$, triglycerides $1.44 \mathrm{mmol} / 1$, fibrinogen $278 \mathrm{mg} / \mathrm{dl}$. Hemophagocytic lymphohistocytosis was diagnosed. Treatment with dexamethasone $40 \mathrm{mg} /$ day for four days and ruxolitinib at $10 \mathrm{mg}$ twice a day was given. We observed a clinical response that permitted cessation of mechanical ventilation and sedation on the $18^{\text {th }}$ day of this 
treatment. A new bone marrow aspirate showed few hemophagocytes and zero blasts. Her karyotype was normal. We classified it as a cytogenetic response in AML. The patient presented a new case of septic shock secondary to systemic aspergillosis and died.

\section{Case 2}

Sixty three year old male without known comorbidities, that presented with anemia and fatigue. He had leukocytosis of $50 \mathrm{x}^{3} / \propto \mathrm{L}$ with $88 \%$ blasts. Bone marrow had $50 \%$ myeloid and monocytic blasts. Cytogenetic risk was favorable due to $t(8 ; 21)$, positive FLT3 TKD and positive monoallelic CEBPA. The same venetoclax + azacytidine scheme was implemented. He presented bilateral pneumonia treated with carbapenem with good answer. Complete response with incomplete blood recovery was found on the $21^{\text {st }}$ day of treatment. On the $32^{\text {nd }}$ day he showed complete blood recovery. In valuation for second cycle, at day +36 , severe neutropenia and thrombocytopenia was detected $\left(\mathrm{Hb} 9.1 \mathrm{~g} / \mathrm{dL}\right.$, granulocytes $0.3 \times 10^{3} / \propto \mathrm{L}$, platelets $22 \times 10^{3} / \propto \mathrm{L}$ ) with bone marrow smear showing hemophagocytic changes without blasts. Triglycerides $0.86 \mathrm{mmol} / \mathrm{L}$, ferritin $5,132 \propto \mathrm{g} /$ $\mathrm{mL}$ and fibrinogen $226 \mathrm{mg} / \mathrm{dL}$. He was treated with dexamethasone 40 $\mathrm{mg}$ /day for 4 days and ruxolitinib $10 \mathrm{mg}$ twice a day with progressive response from fifth day of treatment. Bone marrow was without blasts; 46XY [20], and negative CEBPA mutation. On 23rd day with ruxolitinib he had complete blood recovery and treatment was suspended. The patient remained on venetoclax $100 \mathrm{mg} /$ day adjusted to hematologic toxicity and azacitidine for ten days each 28 days. On October 2019 the patient presented a new episode of aplasia with HLH that responded favorable to ruxolitinib with suspension of azacitidine + venetoclax. He suspended treatment since then and until the last follow up on January 2021 he was in complete cytogenetic remission.

\section{Case 3}

Thirty six year old male, without medical history record. $\mathrm{He}$ started in July 2016 with diagnosis of chronic myeloid leukemia with leukocytosis of 143,000, splenomegaly and Philadelphia chromosome positive and started treatment with dasatinib on August 2016. On May 2018 he progressed to blast phase with $45 \%$ myeloid blasts.
BCR/ABL P210 was found positive in $40.7 \%$. He started the same scheme of azacitidine and venetoclax and a change of dasatinib to nilotinib $400 \mathrm{mg}$ twice a day. On the $11^{\text {th }}$ day of treatment blasts in blood were not observed, marrow blasts reduced to $6 \%$ and $\mathrm{BCR} /$ ABL P210 reduced to $19.5 \%$. He later presented neutropenic fever with livedo reticularis and treatment with meropenem was initiated. Procalcitonin was negative and a bone marrow aspirate was performed detecting hemophagocytic changes. Ferritin $4942 \mathrm{ng} / \mathrm{ml}$, C- reactive protein (CRP) $197 \mathrm{mg} / \mathrm{L}$. Ruxolitinib at $10 \mathrm{mg}$ twice a day was started with clinical improvement at the $4^{\text {th }}$ day of treatment. Due to this improvement venetoclax was reinitiated with fever at the first day of retreatment associated to HLH; dexamethasone was initiated with etoposide. A new bone marrow aspirate was performed showing hypocellularity, negative minimal residual disease and BCR/ ABL detectable but unquantified. The patient developed neutropenic enterocolitis managed successfully with meropenem, amikacin, liposomal amphotericin and IV immunoglobulin. The last BCR/ABL was qualitative and quantitative undetected. On October 2016 the patient presented hematology recovery. He continued with venetoclax $400 \mathrm{mg} /$ day until he received a stem cell transplant of a matched sibling donor.

\section{Discussion}

Here we present three cases of hemophagocytic lymphohistocytosis after the treatment of with venetoclax + azacitidine in two de novo and one secondary AML. In our research we didn't find any report on this kind of reactions nevertheless there are ongoing clinical trials with this scheme as some other trials with venetoclax in combination with distinct drugs. ${ }^{2}$ The first released clinical study of venetoclax in AML was a phase 2 in relapsed/refractory leukemia released in 2014 that showed an overall response in 19\%; the highest dose was $800 \mathrm{mg} /$ day and no tumor lysis syndrome was reported; febrile neutropenia was reported in $31 \% .{ }^{5}$ Due to its response as monotherapy, combination regimens have been developed. A phase $1 \mathrm{~b}$ study of venetoclax with hypomethylating agents was done in 145 elderly patients. The most serious adverse events were also febrile neutropenia and grade 3-4 myelosuppression with no tumor lysis. ${ }^{4}$

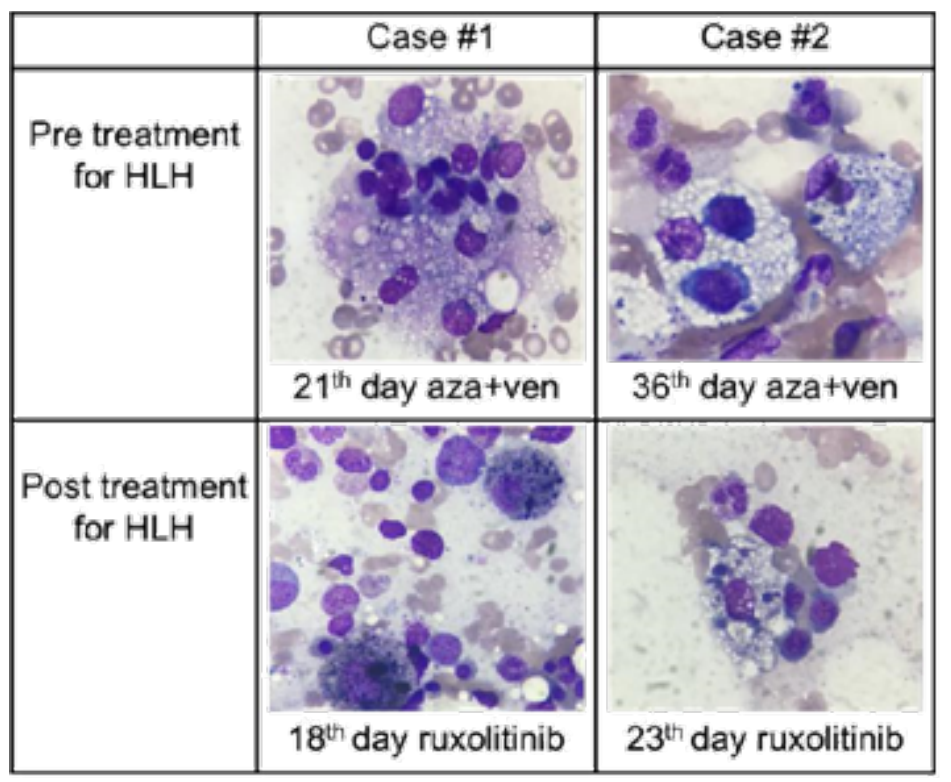

Figure I Images of hemophagocytosis in patients I and 2.

The hemophagocitosis found at the initial syndrome are exposed on the first line while in the second line there is showed the hemophagocytes decrease after treatment with ruxolitinib. Images from bone marrow smear in H\&E stain observen on a Cari Zeiss Microscopy Primo Star. Original Magnificarion x I00. 
Because of high efficiency in the apoptotic pathways ${ }^{1}$ in cells of rapid replication as myeloid blast it is hard to believe that there is no lysis produced by azacitidine + venetoclax in AML. ${ }^{4,5}$ suspicion is that given the physiopathology of this disease, venetoclax in synergy with azacitidine can produce enough proinflammatory cytokines in the medullar niche that subsequently induce hemophagocytosis and its consequential complications. Further studies must be done that could demonstrate what are the factors involved in the development of hemophagocytosis on this group of patients. As shown in these three cases and due to the great efficacy of this scheme we expect to see more patients with this adverse event. We think that reports like this one will help the knowledge of this scheme and learn how to detect and manage its adverse events better.

It is also important to mention the favorable response of hemophagocytosis to ruxolitinib, being reported just in case reports and small series ${ }^{6,7}$ Here we present three cases of favorable response of this drug to help reduce the proinflammatory pathways in secondary hemophagocytosis.

\section{Conflicts of interest}

Roberto Ovilla has performed paid conferences for ABBVIE, JANSSEN, AMGEN, NOVARTIS and TAKEDA. The rest of authors have no disclosures. There was no industry relation during this study.

\section{Acknowledgments}

None.

\section{References}

1. Stilgenbauer S, Eichhorst B, Schetelig J, et al. Venetoclax in relapsed or refractory chronic lymphocytic leukaemia with $17 \mathrm{p}$ deletion: a multicentre, open-label, phase 2 study. Lancet Oncol. 2016;17(6):768-778.

2. Konopleva M, Letai A. BCL-2 inhibition in AML: an unexpected bonus? Blood. 2018;132(10):1007-12.

3. Seymour JF, Ma S, Brander DM, Choi MY, et al. Venetoclax plus rituximab in relapsed or refractory chronic lymphocytic leukaemia: a phase 1b study. Lancet Oncol. 2017;18(2):P230-240.

4. DiNardo CD, Pratz KW, Letai A, et al. Safety and preliminary efficacy of venetoclax with decitabine or azacitidine in elderly patients with previously untreated acute myeloid leukaemia: a non-randomised, openlabel, phase 1b study. Lancet Oncol. 2018;19(2):216-228.

5. Konopleva M, Pollyea DA, Potluri J, et al. Efficacy and Biological Correlates of Response in a Phase II Study of Venetoclax Monotherapy in Patients with Acute Myelogenous Leukemia. Cancer Discov. 2016;6(10):1106-1117.

6. Slostad J, Hoversten P, Haddox CL, et al. Ruxolitinib as first-line treatment in secondary hemophagocytic lymphohistiocytosis: A single patient experience. Am J Hematol. 2018;93(2):E47-E49.

7. Sin JH, Zangardi ML. Ruxolitinib for secondary hemophagocytic lymphohistiocytosis: First case report. Hematol Oncol Stem Cell Ther 2019;12(3):166-170. 